\title{
Regionalização e Análise da Tendência da Precipitação do Rio Grande do Norte Associados a Padrões de TSM
}

\author{
Ana Cleide Bezerra Amorim ${ }^{1}$ (D), Paulo Sérgio Lucio ${ }^{1}$, Cláudio Moisés Santos e Silva ${ }^{1}$, \\ Daniele Tôrres Rodrigues ${ }^{2}$, Francisco Agustinho de Brito Neto ${ }^{3}$ \\ ${ }^{1}$ Programa de Pós-Graduação em Ciências Climáticas (PPGCC), Universidade Federal do Rio \\ Grande Norte, Natal, RN, Brasil. \\ ${ }^{2}$ Universidade Federal do Piaui, Teresina, PI, Brasil. \\ ${ }^{3}$ Instituto Nacional de Pesquisas Espaciais, Cachoeira Paulista, SP, Brasil.
}

Recebido em: 6 de Maio de 2019 - Revisado em: 11 de Setembro de 2019 - Aceito em: 23 de Outubro de 2019

\begin{abstract}
Resumo
A precipitação apresenta elevada variabilidade espacial e temporal, a exemplo do Nordeste do (NEB), que é caracterizado por longos períodos de secas e por episódios de chuva excessiva, que interferem no gerenciamento de recursos hídricos. Especificamente o Estado do Rio Grande do Norte $(\mathrm{RN})$, localizado na região oriental do NEB, a estação chuvosa ocorre em dois períodos. Neste contexto, o objetivo principal deste estudo é definir áreas homogêneas de precipitação (AHP) do Estado do RN, investigar tendências lineares e mudanças em que as mesmas ocorreram na estação chuvosa. Utilizaram-se acumulados de precipitação da quarta versão do produto da Universidade de Delaware para o período de 1900 a 2014. Para identificar as AHP utilizou-se a análise de agrupamento. As tendências foram estimadas pelo teste de Mann-Kendall. As quebras de estrutura, pelo método de Pettit. Os resultados indicam três AHP: Oeste, Seridó e Litoral. A estação chuvosa no Oeste e Seridó concentra-se entre fevereiro a maio; no Litoral, entre abril e julho. As tendências são identificadas apenas no Litoral com valor-p $<0,05$, um aumento em sua média foi observado a partir de 1964 e isso, aparentemente, está associada aos modos de variabilidade do Atlântico.
\end{abstract}

Palavras-chave: Mann-Kendall, Pettitt, tendência positiva, estação chuvosa.

\section{Regionalization and Trend Analysis of Precipitation in Rio Grande do Norte Associated with SST Patterns}

\begin{abstract}
Precipitation presents high spatial and temporal variability, as in Northeast Brazil (NEB), which is characterized by long periods of droughts and episodes of excessive precipitation, which influences the water resources management. Specifically, Rio Grande do Norte (RN) state located in the eastern of NEB, the rainy season occurs in two periods. In this context, the main objective of this study is to define homogeneous areas of precipitation (HAPs) in the RN state to investigate linear trends and changes in which they occurred in the rainy season. It was used the accumulated precipitation from the fourth version of data from the University of Delaware, for the period 1900 to 2014. To identify the HAPs was used the cluster analysis. Trends were estimated by the Mann-Kendall test. The structural breaks was made by the Pettitt method. The results indicate three HAPs in the RN state: West, Seridó and Coast. The rainy season in the West and Seridó is concentrated between February and May; on the coast, between April and July. Trends are identified only on the coast with p-value $<0.05$, an increase in their mean was observed from 1964 and this is apparently associated with the modes of Atlantic variability.
\end{abstract}

Keywords: Mann-Kendall, Pettitt, positive trend, rainy season.

Autor de correspondência: Ana Cleide Bezerra Amorim. email: anacleide23@gmail.com. 


\section{Introdução}

Existe uma vasta literatura científica acerca da ocorrência de precipitação no Nordeste do Brasil (NEB) (Yamazaki e Rao, 1977; Zhou e Lau, 2001; Carvalho et al., 2002, 2004; Ferreira e Mello, 2005; Souza e Cavalcanti, 2009; Reboita et al., 2010; Oliveira et al., 2013; 2016; Rodrigues et al., 2019; 2020), que é caracterizado por apresentar longos períodos de seca (Marengo et al., 2011; Costa et al., 2015; Dos Santos et al., 2019) e ocorrencias de eventos de precipitação intensa (Costa et al., 2015; Oliveira et al., 2017; Da Silva et al., 2019; Rodrigues et al., 2020). O NEB engloba os Estados do Maranhão, Piauí, Ceará, Rio Grande do Norte, Paraíba, Pernambuco, Alagoas, Sergipe e Bahia, totalizando uma área de aproximadamente $1.558 .196 \mathrm{~km}^{2}$. A região apresenta alta variabilidade espacial e temporal da distribuição de chuvas (Moura e Shukla, 1981; Zhou e Lau, 2001; Oliveira et al., 2017; Rodrigues et al., 2019), com acumulados anuais inferiores a $500 \mathrm{~mm}$ em regiões semiaridas e superiores a $1500 \mathrm{~mm}$ em regiões costeiras (Oliveira et al., 2017; Rodrigues et al., 2019). A principal estação chuvosa do NEB ocorre entre março e junho, enquanto o período seco, em grande parte do NEB, ocorre entre agosto e outubro (Rao et al. 1993; Rodrigues et al., 2019).

Dentre os Estados que englobam o NEB, Pernambuco e Rio Grande do Norte (RN) são os que possuem maior extensão de território com clima semiárido (Bsh), respectivamente, cerca de 61,4, e 61,2\% (Alvares et al., 2013). Os Relatórios Científicos do IPCC (AR4 e AR5) expõem o semiárido do Brasil como uma das regiões do globo que são mais afetadas e sensíveis às mudanças climáticas atuais e futuras (Marengo, 2014).

O clima semiárido é predominante na região cobrindo desde áreas costeira do RN em cerca de $150 \mathrm{~km}$ da região litorânea, maior parte do Planalto da Borborema no estado da Paraíba, onde é considerado um dos lugares mais seco do Brasil (o vale do rio Paraíba) semelhante a Paulo Afonso na Bahia que também foi considerado com essa característica (Alvares et al., 2013). Além dos estados do Pernambuco, Ceará com regiões próximas a costa, Alagoas e Sergipe com 14,9 e 13,5\% do território de Bsh. Em contrapartida, apenas o estado do Maranhão não apresenta clima Bsh (Alvares et al., 2013).

Apesar da ocorrência de secas ser uma característica marcante do clima do NEB; diversos sistemas meteorológicos favorecem a precipitação na região ocasionando máximos de precipitação anuais que variam de 300 a $2000 \mathrm{~mm}$ (Rodrigues et al., 2019). Entre esses principais sistemas pode-se citar a Zona de Convergência Intertropical (ZCIT) (Zhou e Lau, 2001; Souza e Cavalcanti, 2009), os Vórtices Ciclônicos de Altos Níveis (VCAN) (Kousky e Gan, 1981; Coutinho et al., 2010), os Distúrbios Ondulatórios de Leste (DOL) (Yamazaki e Rao, 1977; Torres e Ferreira, 2011; Gomes et al., 2015), Linhas de Instabilidade (LI) (Ferreira e Mello, 2005), Complexos Convectivos de Mesoescala (CCM) (Souza et al., 1998; Fedorova et al., 2009; Lyra et al., 2015), Sistemas Frontais (SF) (Kousky, 1979; Alves e Kayano, 1991) e a Zona de Convergência do Atlântico Sul (ZCAS) (Chaves e Cavalcanti, 2001; Carvalho et al., 2002; Carvalho et al., 2004).

A ZCIT é o principal sistema que favorece a precipitação na região NEB. Atua entre as estações de verão e outono austral (Zhou e Lau, 2001), principalmente, no setor Norte do NEB (NNEB), em que em anos chuvosos pode atingir até $5^{\circ} \mathrm{S}$ (Melo et al., 2009). Os VCANs atuam no verão austral com máxima predominância no mês de janeiro (Kousky e Gan, 1981; Coutinho et al., 2010). Os DOL ocorrem durante todo ano (Diedhiou et al., 2010) ao longo da costa NNEB e Leste do NEB (LNEB); contudo são responsáveis por elevados índices pluviométricos nos meses de inverno em regiões do LNEB (Ramos, 1975; Amorim et al., 2019; Rodrigues et al., 2020). Os SF e a ZCAS atuam sobre o setor Sul do NEB (SNEB), em especial, nos meses de novembro a janeiro (Ferreira e Melo, 2005).

A precipitação do NEB é afetada por alguns modos de variabilidade em diferentes escalas de tempo, dos oceanos Pacífico e Atlântico. A influência dos modos de alta frequência no clima do NNEB é bem fundamentada na literatura (Souza e Nobre, 1998; Misra e Zhang, 2007). Basicamente, as alterações na circulação de grande escala leste-oeste (célula de Walker) e norte-sul (célula de Hadley) configuram um ambiente favorável ou desfavorável a atuação dos principais sistemas meteorológicos no NNEB, durante a estação de outono austral (Souza e Ambrizzi, 2002). No entanto, vale lembrar que o RN apresenta máximos de precipitação também no inverno.

Quanto aos modos de variabilidade baixa frequência no Atlântico, um dos existentes no setor Norte (Nobrega et al., 2014) é semelhante à Oscilação Decadal do Pacifico (Newman et al., 2016) com fases quente e fria, chamado de OMA (Oscilação Multidecadal do Atlântico) (Enfield et al., 2001). Durante o período de 1925 a 1960, a OMA apresentava-se na fase positiva e negativa entre 1964 a 1995 (32 anos) (Santos et al., 2016), e após 1995 iniciouse a fase positiva (Trenberth e Zhand, 2019).

O segundo modo de variabilidade do Atlântico é caracterizado por centros de ação com fases opostas, um na região da Islândia e o outro na região do Atlântico Norte Central (Açores), Rogers (1984). No inverno do hemisfério norte (NH) é mais evidente, mas é possível a ocorrência nas demais estações do ano. Existe um índice para verificar esta oscilação (North Atlantic Oscilation Index), quando o valor é positivo, os centros de alta subtropical do Atlântico Norte e a baixa da Islândia apresentam-se anomalamente mais intensos. Justamente na variabilidade da alta subtropical do Atlântico Norte (ASAN) que se deve à influência em relação à precipitação da região NEB. Namais (1972) foi o trabalho pioneiro entre a precipitação no NEB e o NAO mostrando que anos chuvosos advêm 
durante a fase positiva do NAO. A fase negativa do NAO prevaleceu da década de 1950 até meados da década de 70 . A partir do final da década de 1970 a 1990, NAO positivo predominou.

Especificamente, o RN encontra-se na área de atuação da ZCIT. No entanto, o clima do RN apresenta na faixa leste do RN, máximos de precipitação durante as estação do outono (março, abril e maio - MAM) referente a esse sistema, além da estação do inverno (junho, julho e agosto - JJA) austral. Estudos destacam fenômenos no inverno austral da região do NEB, que se aplica ao RN: a ocorrência de DOL, que se propagam para oeste sobre o Oceano Atlântico (Reboita et al., 2010; Torres e Ferreira, 2011; Santos et al., 2012; Kouadio et al., 2012; Gomes et al., 2015; Hounsou et al., 2015; Amorim et al., 2019). Além do DOL, a influência de brisa, especialmente, no período noturno, referente à formação de uma zona de convergência entre a brisa terrestre e o escoamento em baixos níveis (Kousky, 1979) e a variabilidade da Alta Subtropical do Atlântico Sul (ASAS) (Rao et al., 1993). Rao et al. (1993) sugerem que esse sistema é o principal mecanismo de transporte de vapor de água nos primeiros níveis da atmosfera.

O estado do RN apresenta faixa litorânea ao norte e a leste, e a maior população (877,662 habitantes) habita na capital Natal, que se encontra no litoral oriental do estado e ocupa uma área de $167,264 \mathrm{~km}^{2}$ (IBGE, 2014). Recentemente, resultados mostraram evidências que eventos extremos de precipitação (EEP) diários apresentou tendência positiva nos últimos anos (Amorim et al., 2019), entre 1986 a 2016, na cidade de Natal. Alguns desses EEP contribuíram para seguidos desastres naturais, em decorrência a existência de áreas de alto risco natural (Moreira et al., 2014; Macedo et al., 2016; Rodrigues et al., 2020) na capital. Além disso, a população da capital também apresenta-se exposta a frequentes alagamentos devido à obras paradas e/ou incompletas do sistema de drenagem da cidade, que são potencializados na ocorrência dos EEP.

Amorim et al. (2019) destacam para a cidade de Natal, em vários anos de El Niños, a variabilidade anual na frequência de EEP diários apresentou-se diferente. Percebe-se que o RN apresenta variabilidadade da precipitação tanto no espaço quanto no tempo (Rodrigues et al., 2020). Sendo assim, estudos que venham a contribuir com informações fundamentais a ações de adaptações às alterações climáticas são de grande valia. Neste contexto, o objetivo principal deste estudo é investigar tendências climáticas em áreas homogêneas de precipitação do estado do RN, em especial, durante a estação chuvosa, além de correlaciona-las a modos de variabilidade dos oceanos Pacífico e Atlântico tropicais. As hipóteses deste trabalho são descritas pelas diferenças espaciais do comportamento da distribuição de chuvas sobre o RN, assim como a influência da variabilidade dos oceanos Pacífico e Atlântico tropicais em relação ao quantitativo da precipitação no RN.

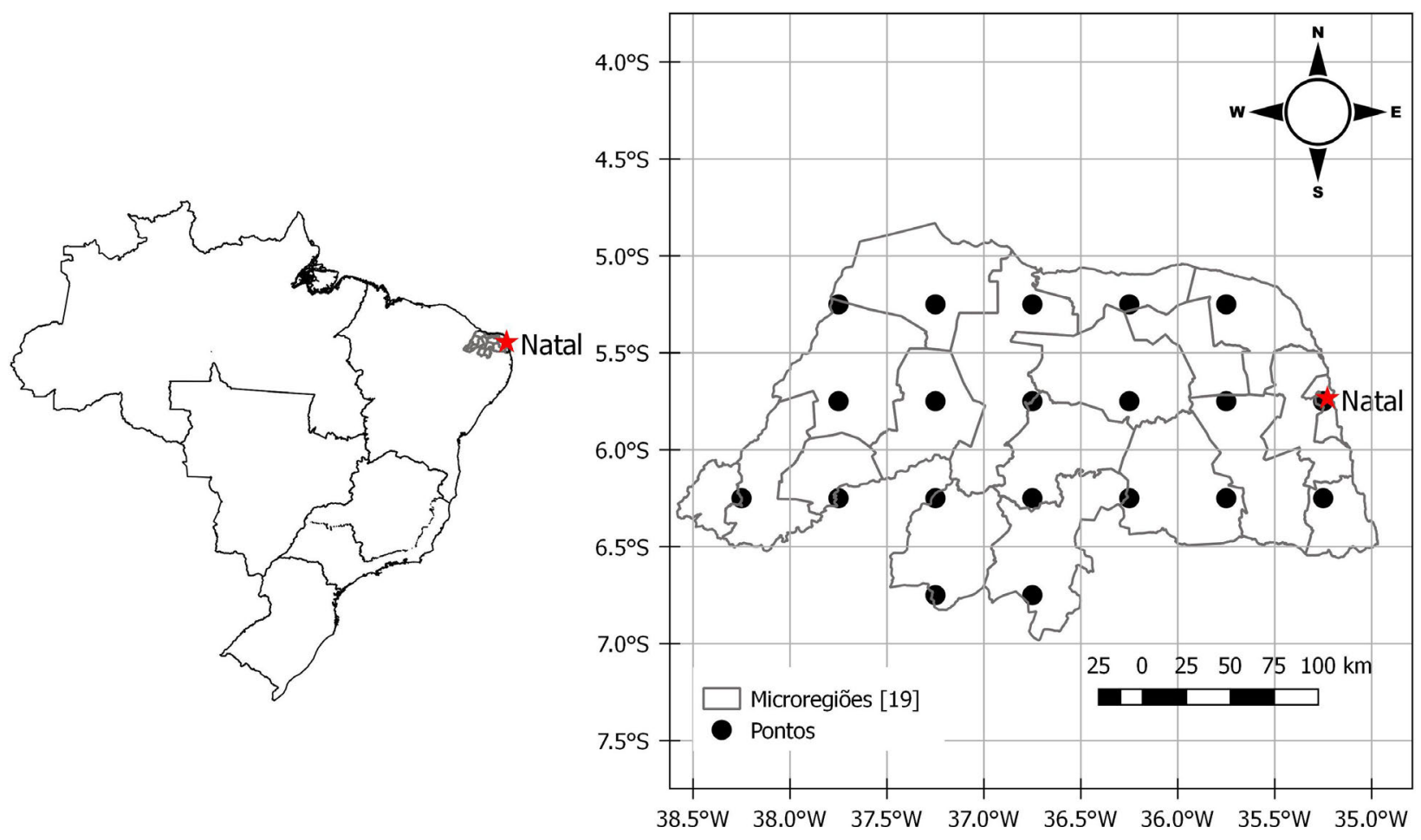

Figura 1 - Mapa de localização do RN e pontos de grade utilizados nas análises. 


\section{Materiais e Métodos}

\subsection{Dados}

Utilizou-se a quarta versão do produto precipitação mensal disponibilizado pela Universidade de Delaware para analisar o clima do RN. Foram obtidos vinte pontos de grade de espaçamento de $0,5^{\circ} \times 0,5^{\circ}$ de latitude por longitude (Fig. 1). O produto apresenta dados interpolados para uma grade regular (Matsuura e Willmott, 2009) e cobre o período de 1900 a 2014. A construção dos dados partiu de múltiplas fontes (Global Historical Climatology Network - GHCN2; Serviço Atmosférico e Ambiental do Canadá; Instituto hidrometeorológico de São PetersburgoRússia cedido por Nikolay Shiklomanov; dados do projeto Greenland Climate Network - GC-Net, Steffen et al. (1996); registros das estações meteorológicas automáticas do Projeto Groenlândia disponibilizados por Charles R. Stearns na Universidade de Wisconsin-Madison; dados diários da Índia do National Center for Atmospheric Research - NCAR; dados de precipitação do continente Africano, Nicholson (2001); dados mensais de precipitação do continente sul-americano, Webber e Willmott (1998); e do Global Surface Summary of Day - GSOD); no entanto, o GHCN2 é a fonte de dados principal do produto.

Utilizaram-se dados de temperatura da superfície do mar (TSM) mensal do National Oceanic \& Atmospheric Administration - Extended Reconstructed Sea Surface Temperature V3b (NOAA ERSST V3b) dispostos em grade horizontal regular de $2,0^{\circ}$ latitude $\times 2,0^{\circ}$ longitude (Smith et al., 2007) para as áreas dos Oceanos Atlântico e Pacífico, no período de 1900 a 2012.

No Pacífico, as áreas tradicionais do Niño $3\left(150^{\circ} \mathrm{W}\right.$ $\left.-90^{\circ} \mathrm{W} / 5^{\circ} \mathrm{S}-5^{\circ} \mathrm{N}\right)$ e Niño $3.4\left(170^{\circ} \mathrm{W}-120^{\circ} \mathrm{W} / 5^{\circ}\right.$ $\left.\mathrm{S}-5^{\circ} \mathrm{N}\right)$ durante os meses de novembro a fevereiro (NDJF) (Trenberth, 1997) e para o Atlântico Tropical Norte $\left(5-20^{\circ} \mathrm{N}, 60-30^{\circ} \mathrm{W}\right)$ e $\mathrm{Sul}\left(0-20^{\circ} \mathrm{S}, 30^{\circ} \mathrm{W}-10^{\circ}\right)$ no trimestre março a maio (MAM).

Também se utilizaram séries temporais dos modos de variabilidade Atlantic Multidecadal Oscillation (AMO) para o período de 1900 a 2014 e North Atlantic Oscillation (NAO) de 1950 a 2014 disponibilizados pelos endereços eletrônicos https://climatedataguide.ucar.edu/climate-data/ atlantic-multi-decadal-oscillation-amo e ftp://ftp.cpc.ncep. noaa.gov/wd52dg/data/indices/, respectivamente.

\subsection{Métodos}

Utilizou-se a análise de agrupamento para identificar áreas homogêneas durante 1900 a 2014. A avaliação do agrupamento realizou-se através do gráfico de Silhueta, desenvolvida por Rousseeuw (1987) e utilizada por diversos autores, Bernard et al. (2013); Santos et al. (2015); Wilcke e Bärring (2016); Mooney et al. (2017); Rahman et al. (2018); Salehnia et al. (2019), em estudos na área de ciências climáticas. Em seguida, investigaram-se as tendências climáticas na quadra chuvosa e possíveis quebras estruturais na série de dados, além de correlações com os índices oceânicos dos Oceanos Pacífico e Atlântico. A correlação cruzada foi utilizada para identificar a defasagem entre os modos AMO e NAO com a precipitação do RN.

\subsubsection{Análise de agrupamento (Cluster)}

A análise de agrupamento é um método multivariado que tem como objetivo separar grupos de acordo com uma medida de dissimilaridade entre eles (Wilks, 2006). Utilizou-se essa técnica para identificar regiões cujo comportamento da precipitação do $\mathrm{RN}$ possa ser considerado homogêneo durante 1900 a 2014. Aplicou-se a técnica aos dados de precipitação mensal (janeiro a dezembro) para vinte pontos de grade do domínio do estado exposto na Fig. 1.

Utilizou-se a distância euclidiana (Eq. 1) como medida de dissimilaridade comumente usadas em aplicações a dados meteorológicos.

$$
d_{i, j}=\left[\sum_{k=1}^{k} W_{k}\left(X_{i, k}-X_{j, k}\right)^{2}\right]^{1 / 2}
$$

em que $X_{i, k}$ e $X_{j, k}$ são as séries temporais para cada ponto de grade comparados dentro do grupo $\mathrm{k} ; \mathrm{W}_{\mathrm{k}}=1, \ldots, k$ é um fator de ponderação e os índices $i$ e $j$ representam os pontos de grade do grupo.

Além da medida de dissimilaridade, utilizou-se o método de agrupamento hierárquico de variância mínima de Ward (Eq. 2) definida segundo a Eq. 2:

$$
\begin{aligned}
W & =\sum_{g=1}^{G} \sum_{i=1}^{n_{g}}\left\|X_{i}-\bar{X}_{g}\right\|^{2} \\
& =\sum_{g=1}^{G} \sum_{i=1}^{n_{g}} \sum_{K=1}^{K}\left(X_{i, K}-\bar{X}_{g, K}\right)^{2}
\end{aligned}
$$

sendo que $\mathrm{G}$ e g representam os grupos de pontos de grade; $X_{i}$ é cada vetor de pontos de grade da variável $\mathrm{K}$; $\bar{X}_{g}$ é a média de todos os pontos de grade do grupo $\mathrm{g}$ da variável $\mathrm{K}$.

Em seguida utilizou-se o gráfico de Silhueta (Rousseeuw, 1987) para averiguar a construção de cada agrupamento (Santos et al., 2015). A Silhueta é a média do valor do contorno de cada observação. Observações agrupadas adequadamente possuem valores próximos de 1 e observações mal agrupadas, valores próximos de -1 . O valor da Silhueta do objeto $\mathrm{X}_{\mathrm{i}}$ (cada observação) é calculado pela Eq. (3): 


$$
S\left(X_{i}\right)=\frac{b\left(X_{i}\right)-a\left(X_{i}\right)}{\max \left\{a\left(X_{i}\right), b\left(X_{i}\right)\right\}}
$$

em que $a\left(X_{i}\right)$ é o comprimento médio de todas as distâncias dentro do mesmo grupo e $b\left(X_{i}\right)$ é comprimento médio de todas distâncias das observações do grupo mais próximo.

A qualidade total do aglomerado pode ser calculada pelo coeficiente de Silhueta médio pela Eq. (4):

$$
\bar{S}=\frac{\sum_{i=1}^{N} S\left(X_{i}\right)}{N}
$$

em que $\mathrm{N}$ é o número total de pontos de grade.

\subsubsection{Análise da tendência}

Utilizou-se para análise da tendência linear o teste não-paramétrico Mann-Kendall (Mann, 1945; Kendall, 1975). Aplicou-se o teste para o acumulado de precipitação do período chuvoso. O uso do teste Mann-Kendall (MK) tem sido comum para mostrar tendências hidrológicas (Santos e Santos e Silva, 2013; Costa et al., 2015; Oliveira et al., 2017), além de ser um método adequado no uso de análises de mudanças climáticas (Goossens e Berger, 1986). Utilizou-se o teste Pettitt (Pettitt, 1979) para identificar possível ponto único de mudança no acumulado de precipitação da estação chuvosa durante o período de estudo. Os níveis significância foram adotados de $1 \%$ e $5 \%$.

\section{Resultados e Discussão}

Os resultados partiram da análise de agrupamento sugerindo três áreas homogêneas (Fig. 2) para o estado do RN: Oeste, Seridó (central) e Litoral. Em seguida, aplicaram-se os testes para série temporal da estação chuvosa de cada grupo, além da investigação aos modos de variabilidade dos Oceanos Pacífico e Atlântico.

\subsection{Regiões homogêneas}

Os resultados da análise de agrupamento são exibidos para duas (Fig. 2a) e três regiões (Fig. 2d) homogêneas, a preferência de três regiões (Fig. 2d) são mais consistentes com a distribuição espacial da climatologia de precipitação, concordando com a análise prévia de Santos e Silva et al. (2012). Além disso, diferenças significativas (valor-p $<0,01$ ) foram obervadas no acumulado de precipitação nos meses da estação chuvosa, entre os grupos encontrados do Semiárido (Fig. 2f).

Os resultados da qualidade dos agrupamentos exibem os coeficientes de Silhueta $\left(S\left(X_{i}\right)\right)$ de cada grupo diminuíndo ao aumentar a quantidade de grupos. Por exem- plo, para dois grupos (Fig. 2b) os valores apresentaram-se acima de 0,60 $\left(S\left(X_{i}\right)=0,66\right.$ no Litoral e $S\left(X_{i}\right)=0,62$ no Semiárido). Para três grupos (Fig. 2e) os valores $S\left(X_{i}\right)$ [U1] diminuem para 0,45 e 0,32 , respectivamente. $O$ grupo do Litoral apresentou valor $S\left(X_{i}\right)$ acima de 0,6 nas análises de agrupamento (Fig. 2b e 2e).

Valor da qualidade total do $\bar{S}$ semelhante $(\bar{S}=0,45)$ foi encontrado em três grupos para a região Amazônica, no entanto, foram necessários seis grupos para caracterizar a variabilidade da precipitação por se tratar de uma região com expressiva extensão territorial, além dos diferentes regimes e intensidades da precipitação (Santos et al., 2015). No RN, apenas com três grupos foi suficiente demonstrar os regimes de precipitação existentes no clima do estado.

Apesar da análise com dois grupos indicar maiores valores da qualidade total, $\bar{S}=0,66$ (ver Fig. 2b), a análise com três grupos ainda apresenta valores adequados, $\bar{S}=0,40$ (Fig. 2e). Deste modo, deu-se continuidade a análise de agrupamento para três grupos, e nessa fase a variabilidade espacial da precipitação demonstrada na literatura (Santos e Silva et al., 2012) foi evidenciada nos resultados (Fig. 2d). A região do Litoral e Oeste apresentaram chuvas mais abundantes que a região do Seridó (Fig. 2f), apresentando acumulado médio anual de 1284, 731 e $566 \mathrm{~mm}$, respectivamente.

O ciclo anual médio dos grupos é exibido na Fig. 3, nos quais se observam a estação chuvosa dos grupos do Semiárido durante os meses de fevereiro a maio apresentando um acumulado médio de $561 \mathrm{~mm}$ para o Oeste (Fig. 3a) e $387 \mathrm{~mm}$ para o Seridó (Fig. 3b), concordando com Marengo et al. (2011). O grupo Litoral apresentou a estação chuvosa nos meses de abril a julho com acumulado médio de $772 \mathrm{~mm}$ (Fig. 3c). A estação seca compreende períodos semelhantes entre os grupos, no entanto, o grupo do Oeste apresenta o período de estiagem entre os meses de agosto a novembro com precipitação média de $20 \mathrm{~mm}$, e os grupos do Seridó e Litoral a partir de setembro até dezembro com acumulados médios de $31 \mathrm{e}$ $105 \mathrm{~mm}$, respectivamente.

A presença da ZCIT na região NEB é o principal sistema meteorológico responsável pela ocorrência de precipitação da estação chuvosa na região do $\mathrm{RN}$, principalmente, nos grupos do Semiárido (Oeste e Seridó) e nos meses de março e abril do grupo do Litoral. A partir de maio e inverno autral, destaca-se a influência dos distúrbios ondulatórios de leste (Gomes et al., 2015), principalmente, no mês junho no Litoral que apresenta o máximo de precipitação média de $210 \mathrm{~mm}$. Além disso, eventos de precipitação intensa durante o inverno austral foram associados a uma circulação anticiclônica anômala que predomina na região em $850 \mathrm{hPa}$ (Oliveira et al., 2013), onde os ventos dessa circulação convergem com vento do Oceano Atlântico, criando uma área de confluência transportando umidade para o grupo do Litoral. 
a)
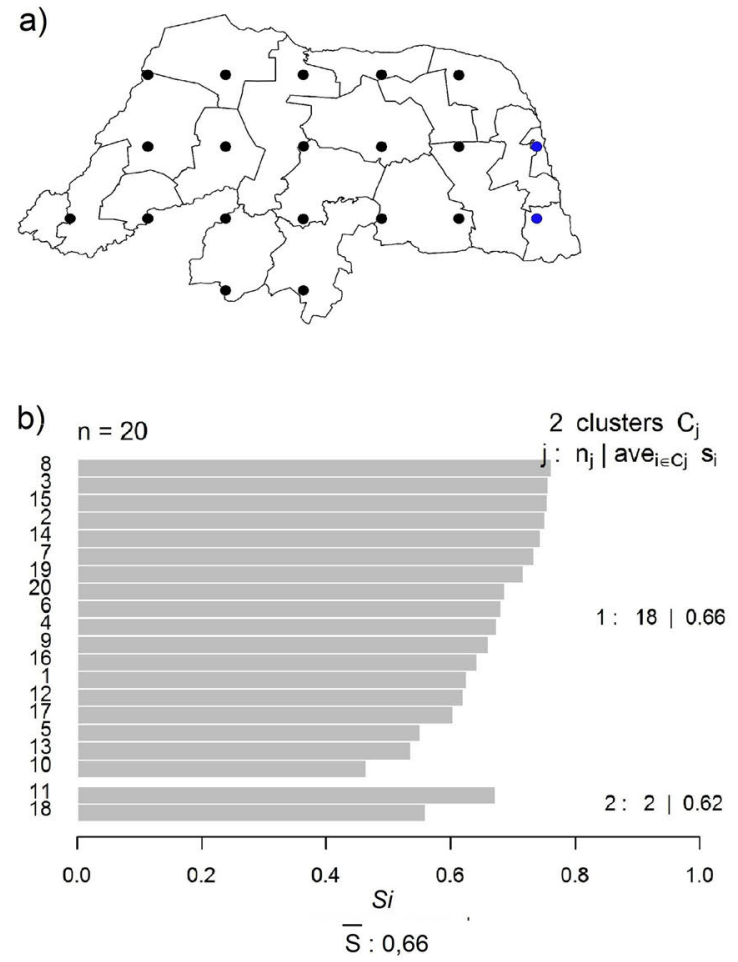

c)

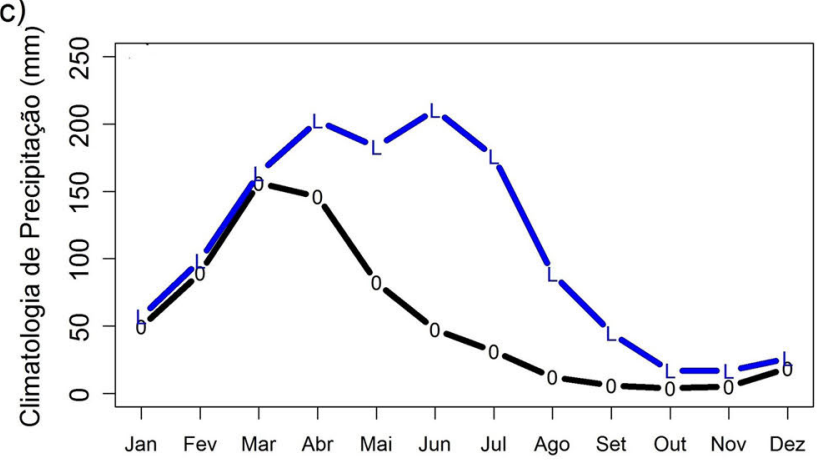

d)
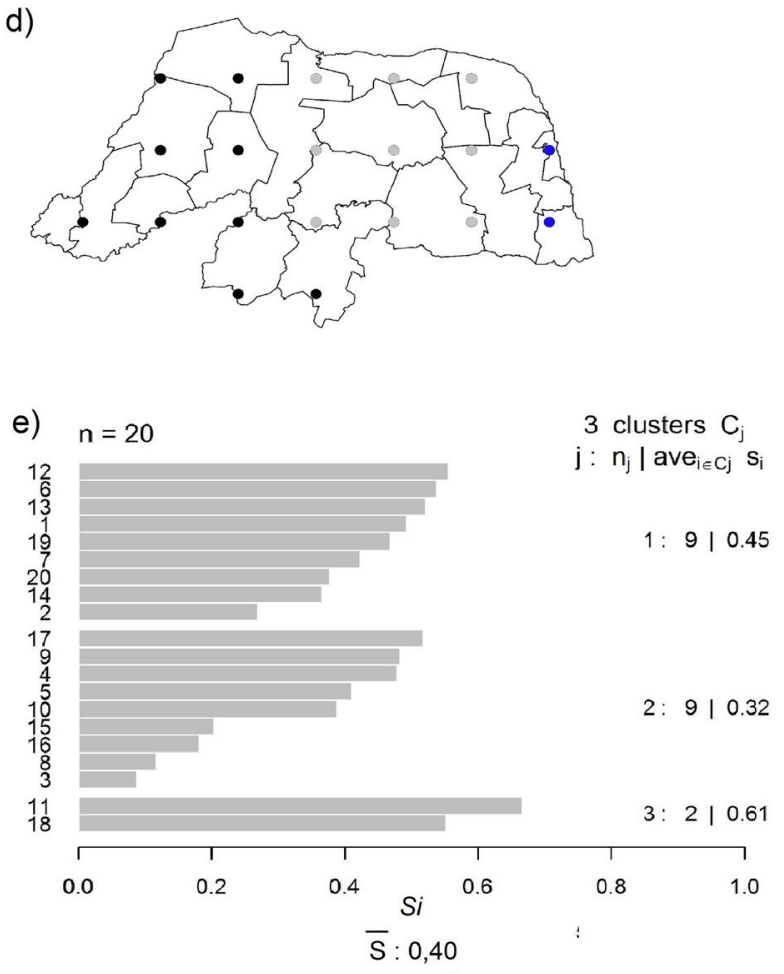

f)

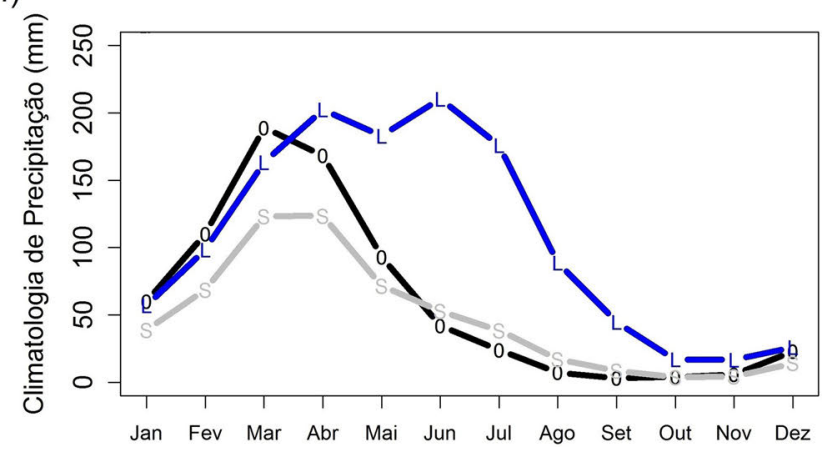

Figura 2 - Resultados da análise de agrupamento para o RN no período de 1900 a 2014 para dois e três grupos (ilustrações superiores e inferiores, respectivamente): (a) - (d) distribuição espacial das áreas homogêneas; (b) - (e) coeficientes de silhueta (qualidade dos agrupamentos); (c) - (f) ciclo anual de precipitação em milímetros, as letras $\mathrm{O}, \mathrm{S}$ e L representam as regiões Oeste, Seridó e Litoral.
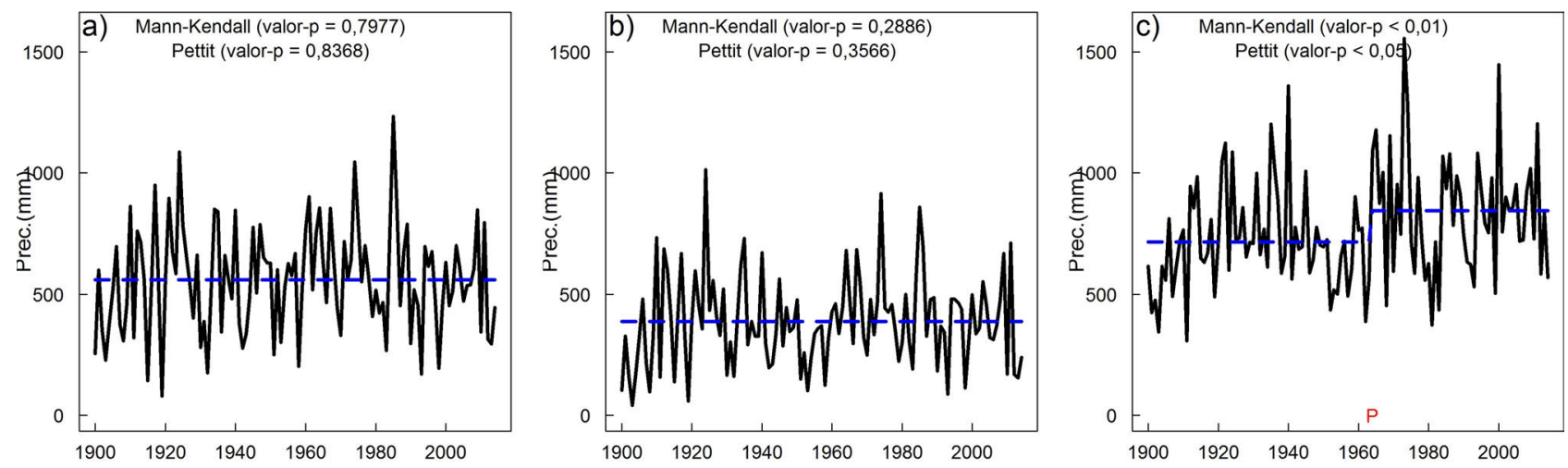

Figura 3 - Série temporal do acumulado de precipitação da quadra chuvosa das áreas homogêneas de precipitação do RN no período de 1900 a 2014: (a) Oeste, (b) Seridó e (c) Litoral. A linha tracejada azul representa o valor médio da precipitação. 
Durante o período de estiagem da costa norte do LNEB, que envolve o grupo do Litoral foi associado ao predomínio de VCANs (Oliveira et al., 2013). Esses sistemas meteorológicos foram associados eventos de precipitação intensas durante a estação seca da costa atuando a partir de novembro e durante o verão austral.

\subsection{Análise de tendência linear}

Aplicou-se o teste da tendência para o acumulado de precipitação do período chuvoso de 1900 a 2014 em cada grupo e os resultados são apresentados na Fig. 3. A análise da tendência não mostrou resultados significativos nas regiões do Semiárido (Oeste e Seridó). Em contrapartida, o Litoral sofreu um acréscimo de $130 \mathrm{~mm}$ na estação chuvosa com nível de significância de $1 \%$. O teste de Pettitt (P) identificou uma mudança significativa (valor-p $<0,05)$ na série a partir da década de 60. O acumulado de precipitação da estação chuvosa até meados dessa década não apresentava mudança no valor médio (linha tracejada azul, Fig. 3c), partir de 1964 a estação chuvosa passou a apesentar valores mais elevados refletindo no valor médio de 714 para $844 \mathrm{~mm}$ (Fig. 3c).

A precipitação do NEB é afetada por alguns modos de variabilidade em diferentes escalas de tempo, dos oceanos Pacífico e Atlântico. A mudança no acumulado de precipitação da estação chuvosa do Litoral do RN pode ser atribuída algum desses modos de variabilidade. Nóbrega et al. (2014) observaram que a precipitação extrema de regiões que se aproximam da área litorânea no estado da Paraíba, como as regiões da Borborema/Agreste e Litoral confrontadas ao Sertão, destacam-se apenas com os modos do Atlântico.

Este comportamento que Nóbrega et al. (2014) observaram de regiões que se aproximam da área litorânea foi evidenciado nos dados da estação chuvosa do $\mathrm{RN}$ (Tabela 1) as tradicionais áreas do Pacífico (Niño3 e Niño3.4, durante os meses de novembro a fevereiro) e Atlântico Tropical Norte e Sul (trimestre março a maio).

Os resultados com modos de alta frequência mostraram correlações com significância estatística (valor-p < $0,01)$ entre as áreas do Semiárido (Oeste e Seridó) e as tradicionais áreas dos oceanos (Tabela 1). Em contrapartida, na área do Litoral a correlação apresentou significância estatística (valor-p < 0,01) apenas com Atlântico Tropical Sul - ATLS (Tabela 1), resultado semelhante foi observado por Amorim et al., 2014.

Após o grupo do Litoral apresentar correlação significativa (valor-p < 0,01) com ATLS, e estudos mostrarem que esse setor do Atlântico é reconhecido por apresentar uma variabilidade independente de outras regiões oceânicas (Chang et al., 1998; Czaja et al., 2002; Barreiro et al. 2004, 2005; Trzaska et al. 2007), diferentemente do ATLN que é fortemente influenciado pelo Pacífico Equatorial sendo positivamente correlacionado com o índice Niño 3 (Enfield e Mayer 1997; Alexander e Scott 2002; Huang et al., 2005a), investigou-se a tendência linear durante o trimestre MAM para o ATLS. Aplicou-se o teste da tendência para as anomalias de TSM (ATSM) do ATLS durante o período MAM de 1900 a 2012, e os resultados são apresentados na Fig. 4.

A análise da tendência mostrou um aumento nos valores das ATSM com significância estatística (valor-p < $0,01)$ para região do ATLS. A região que apresentou correlações significativas com o litoral do RN sofreu um acréscimo de $0,6{ }^{\circ} \mathrm{C}$ entre o período de 1900 a 2012 (Fig. 4a). O teste de Pettitt (P) identificou uma mudança significativa (valor-p $<0,01$ ) na série a partir da década de 60 (Fig. 3c). As ATSM do período MAM apresentava valor médio de $-0,73{ }^{\circ} \mathrm{C}$ (linha tracejada azul, Fig. 4a), partir de 1960 ATSM passou a apresentar valores mais elevados refletindo no valor médio de $0,11^{\circ} \mathrm{C}$.

A mesma análise se repetiu para o período a partir de 1960 a 2012 (Fig. 4b) para o trimestre MAM do ATLS. Os resultados mostram um aumento significativo (valor-p < $0,01)$ de $0,2^{\circ} \mathrm{C}$ das ATSM a partir de meados da década de 80 (Fig. 4b). As ATSM apresentada anteriormente exibem um valor médio de $-0,3{ }^{\circ} \mathrm{C}$, a partir de 1985 ATSM passa a apesentar valor médio de $0,1^{\circ} \mathrm{C}$ (Fig. 4b).

A influência que os modos de variabilidade dos oceanos apresentam no clima, alguns não ocorrem de maneira simultânea. $\mathrm{O}$ resultado da correlação sem defasagem de tempo entre os dados OMA e NAO com a precipitação na escala mensal confirma esse comportamento, apesar de apresentarem significância estatística com valores-p $<0,01$, os valores dos coeficientes de correlação apresentam-se abaixo de 0,2 para os três grupos do RN.

Tabela 1 - Coeficiente de correlação com significância estatística de 1\% entre o período de 1900 a 2012 entre a precipitação da estação chuvosa das áreas do Oeste (FMAM), Seridó (FMAM) e Litoral (AMJJ) e as anomalias de TSM (ATSM) das tradicionais áreas dos Nino3, Nino3.4, Atlântico tropical Norte e Sul.

\begin{tabular}{cccr}
\hline Anomalias de TSM & Oeste & Seridó & Litoral \\
\hline Nino 3 & $-0,35(<0,01)$ & $-0,29(<0,01)$ & $0,03(0,7718)$ \\
Nino 3.4 & $-0,35(<0,01)$ & $-0,28(<0,01)$ & $0,01(0,8803)$ \\
Atlântico Norte & $-0,34(<0,01)$ & $-0,26(<0,01)$ & $0,01(0,9228)$ \\
Atlântico Sul & $0,41(<0,01)$ & $0,45(<0,01)$ & $0,49(<0,01)$ \\
\hline
\end{tabular}



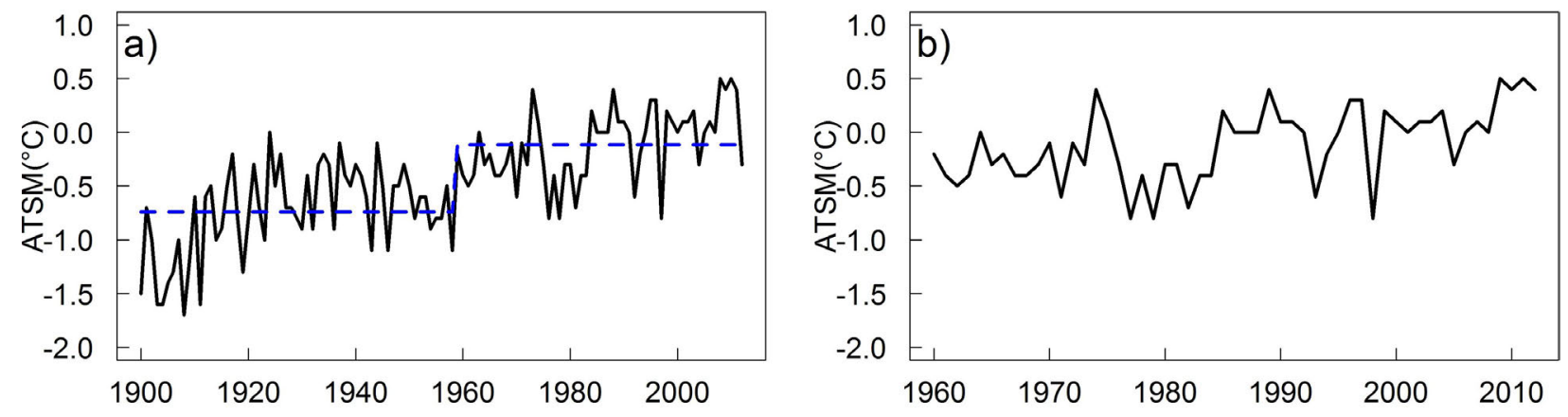

Figura 4 - Série temporal das ATSM do ATLS para o período de MAM no período de 1900 a 2012. A linha tracejada azul representa o valor médio da anomalia: (a) Período de 1900 a 2012 e (d) Período de 1960 a 2012.

A correlação cruzada entre o modo OMA e a precipitação dos grupos do RN exibiram defasagens a partir de um mês até doze meses anteriores. O segundo modo de variabilidade do Attântico (NAO), a correlação cruzada não mostrou resultados satisfatórios.

O modo OMA apresentou correlações significativas com grupos do Semiárido (Oeste e Seridó) apenas nas defasagens entre dois e seis meses que antecedem a estação chuvosa, ou seja, durante julho a dezembro do ano anterior, as correlações foram $-0,38$ com o grupo Oeste e $-0,44$ com Seridó). Apesar do resultado mostrar correlações com valores baixos, a relação negativa exibida com dados da precipitação dos grupos do $\mathrm{RN}$ apresentaram significância estatística (valor-p $<0,01$ ). No entanto, o Litoral mostrou correlações de $-0,23$ entre quatro e seis meses que antecederam o período de AMJJ com nível de significância de $10 \%$. A Fig. 5 ilustra a série temporal mensal do OMA e NAO.

O comportamento do Atlântico, em específico o ATLS, pode ter contribuído para mudança no acumulado médio da precipitação do Litoral do RN. Estudos mais detalhados estãos em andamento, além de outros bancos de dados estão sendo explorados para complementar os resultados discutidos neste estudo.

A fase negativa (1964 a 1995) da OMA, além da tendência de aumento das ATSM do ATLS são indícios para mudança no acumulado de precipitação discutida para o Litoral do RN. Paralelo a esses resultados, o cresci-

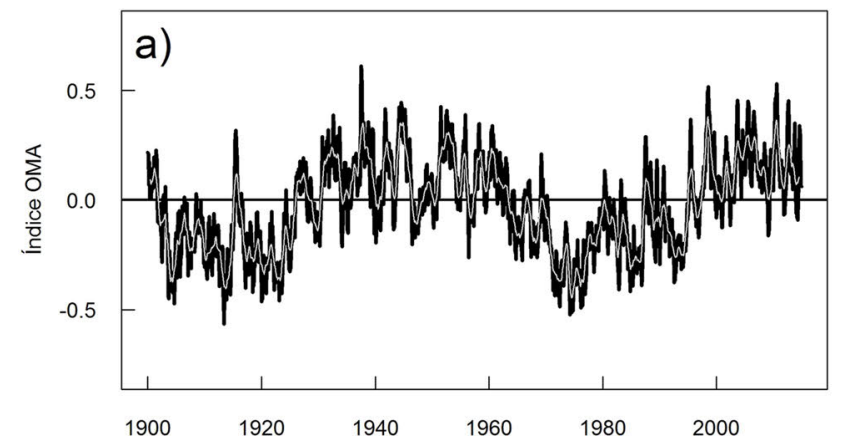

mento populacional e a urbanização são fatores que precisam ser analisados, pois suas influências são poucas discutidas na literatura. E a população do RN foi estimada em 3.474,998 habitantes (IBGE, 2014) e a maior população $(877,662$ habitantes) reside no Litoral, onde se localiza a capital Natal.

\section{Considerações Finais}

Apresentou-se uma avaliação da precipitação do produto de precipitação da Universidade de Delaware. Essa fonte de dados capturou a variabilidade espacial e temporal da precipitação mensal, destacando-se áreas mais secas e mais chuvosas presentes no Estado do RN.

Os resultados da análise de agrupamento foram consistentes com a literatura sugerindo três grupos para o RN: Oeste, Seridó e Litoral. A diferença entre os grupos do Semiárido (Oeste e Seridó) apresentou-se na intensidade da precipitação durante a estação chuvosa.

Os resultados da análise da tendência mostraram evidências que o acumulado de precipitação na região onde se localiza a capital do RN apresentou mudança. Paralelo a esse resultado, o Atlântico Tropical Sul apresentou um aumento nas anomalias de temperatura da superfície na década em que a mudança no acumulado médio da precipitação do grupo do Litoral foi observada. A importante influência exercida pelo Atlântico Tropical

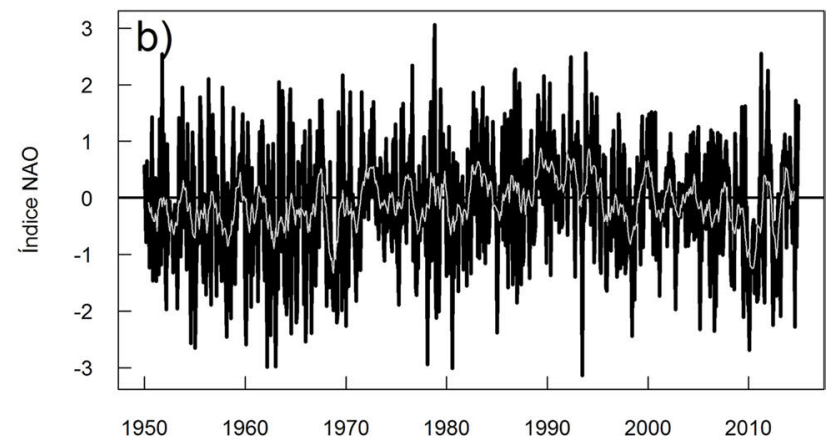

Figura 5 - Índices: (a) da OMA de janeiro de 1900 a dezembro de 2014 e (b) NAO, de janeiro de 1950 a dezembro de 2014. Fonte: NOAA. 
Sul à região litorânea discutida na literatura foi mais uma vez ilustrada nos resultados deste estudo.

Além dos modos de variabilidade presentes no Atlântico, o crescimento populacional também precisa ser explorado para verificar possíveis contribuições às alterações climáticas nas regiões litorânes, regiões estas que apresentam cada vez mais ocupação de áreas de risco, refletindo em mais desastres naturais nunca antes registrados.

Em geral, os resultados obtidos neste estudo fornecerão o conhecimento prévio do comportamento da precipitação sobre o RN. Espera-se que a subdivisão espacial definida nesse estudo, seja considerada por pesquisadores que desejam estudar a precipitação sobre o RN, uma vez que as sub-regiões aqui definidas caracterizam a variabilidade espacial presente no Estado de acordo com a precipitação. Além disso, os resultados deste estudo foram capazes de identificar tendência positiva, estatisticamente significativa (valor-p $<0,01$ ), dos valores anuais de precipitação acumulada sobre o litoral do RN. Este resultado foi associado com a tendência positiva observada sobre as anomalias de TSM no ATLS, que foi estatísticamente significante (valor-p $<0,01$ ) para o período de MAM.

Espera-se que os resultados deste estudo sejam benéficos para amparar a formulação de políticas públicas para minimizar a vulnerabilidade da população do RN, sobretudo aquela que vive em áreas de risco, por meio da prevenção e mitigação de eventos catastróficos que podem ocorrer devido ao excesso ou escassez de chuvas.

\section{Agradecimentos}

Os autores desejam agradecer ao PNPD/Capes (Programa Nacional de Pós Doutorado/Aperfeiçoamento de Pessoal de Nível Superior) pela bolsa do Pós-doutorado concedida à primeira autora.

\section{Referências}

ALEXANDER, M.; SCOTT, J. The influence of ENSO on air sea interaction in the Atlantic. Geophysical. Research. Letters. v. 29, n. 14, p. 1701-1704, 2002.

ALVAREZ, C.A.; STAPE, J.L.; SENTELHAS, P.C.; DE MORAES GONÇALVES, J. L.; SPAROVEK, G. Köppen's climate classification map for Brazil. Meteorologische Zeitschrift, v. 22, n. 6, p. 711-728, dec. 2013.

ALVES, J.M.B.; KAYANO, M.T. Estudo preliminar da precipitação no sul do Ceará durante a pré-estação chuvosa. Boletim Climanálise, v. 6, n. 2, p. 41-50, 1991.

ALVES, J.M.B.; REPELLI, C.A. Variabilidade pluviométrica no setor norte do Nordeste e os eventos El-Niño Oscilação Sul. Revista Brasileira de Meteorologia, v. 7, n. 2, p. 583-592, 1992.

AMORIM, A.C.B.; CHAVES, R.R.; SILVA, C.M.S. Influence of the Tropical Atlantic Ocean's Sea Surface Temperature in the Eastern Northeast Brazil Precipitation. Atmospheric and Climate Sciences, v. 4, n. 5, p. 874-883, 2014.

AMORIM, A. C.B.; SCUDELARI, A.C.; AMARO, V.E.; MATOS, M.F.A. Variabilidade dos extremos de precipitação diária na cidade de Natal, Estado do RN, Nordeste do Brasil. Anuário do Instituto de Geociências, v. 42, n. 2, p. 284-295, 2019.

BARREIRO, M.; CHANG, P.; SARAVANAN, R. Simulated precipitation response to SST forcing and potential predictability in the region of the South Atlantic convergence zone. Climate Dynamics, v. 24, n. 1, p. 105-114, 2005.

BARREIRO, M.; GIANNINI, A.; CHANG, P.; SARAVANAN, R. On the role of the Southern Hemisphere atmospheric circulation in tropical Atlantic variability. Monograph. American Geophysical Union, v. 147, n. 1, p. 143-156, 2004.

BERNARD, E.; NAVEAU, P.; VRAC, M.; MESTRE, O. Clustering of maxima: Spatial dependencies among heavy rainfall in France. Journal of Climate, v. 26, n. 20, p. 79297937, 2013.

CARVALHO, L.M.V.; JONES, C.; LIEBMANN, B. The South Atlantic convergence zone: Intensity, form, persistence and relationships with intraseasonal to interannual activity and extreme rainfall. Journal of Climate, v. 17, n. 1, p. 88-108, 2004.

CARVALHO, L.M.V.; JONES C.; LIEBMANN, B. Extreme Precipitation Events in Southeastern South America and Large-Scale Convective Patterns in the South Atlantic Convergence Zone. Journal of Climate, v. 15, n. 17, p. 2377-2394, 2002.

CHANG, P.; PENLAND, C.; JI, L.; MATROSOVA, L.; LI, H. Prediction of tropical Atlantic sea surface temperature. Geophysical Research Letters, v. 25, n. 8, p. 1193-1196, 1998.

CHAVES, R.C.; CAVALCANTI, I.F.A. Atmospheric circulation features associated with the precipitation variability over the southern Northeast Brazil. Monthly Weather Review, v. 129, n. 10, p. 2614-2626, 2001.

COSTA, M.S.; LIMA, K.C.; ANDRADE, M.M.; GONÇALVES, W.A. Tendências observadas em extremos de precipitação sobre a região Semiárida do Nordeste do Brasil. Revista Brasileira de Geografia Física, v. 08, n. 05, p. 1321-1334, 2015.

COUTINHO, M.D.L.; GAN, M. A.; RAO, V. B. Método objetivo de identificação dos vórtices ciclônicos de altos níveis na região Tropical Sul: validação. Revista Brasileira Meteorologia, v. 25, n. 3, p. 311-323, 2010.

CZAJA, A.; VAN DER VAART. P.; MARSHALL, J. A diagnostic study of the role of remote forcing in tropical Atlantic variability. Journal of Climate, v. 15, n. 22, p. 32803290, 2002.

DIEDHIOU, A.; MACHADO, A.T.; LAURENT, H. Mean Kinematic Characteristics of Synoptic Easterly Disturbances over the Atlantic. Advances in Atmospheric Sciences, v. 27, n. 3, p. 483-499, 2010.

DOS SANTOS, S.R.Q.; DO AMARAL CUNHA, A.P.M.; RIBEIRO-NETO, G.G. Avaliação de dados de precipitação para o monitoramento do padrão espaço-temporal da seca 
no nordeste do Brasil. Revista Brasileira de Climatologia, v. 25, n. 2, p. 80-100, 2019.

ELFIELD, D.B.; MAYER, D.A. Tropical Atlantic SST variability and its relation to El Niño-Southern Oscillation. Journal of Geophysical Research, v. 102, n. C1, p. 929-945, 1997.

ENFIELD, D.B.; MESTAS-NUÑEZ, A.M.; TRIMBLE, P.J. The Atlantic multidecadal oscillation and its relation to rainfall and river flows in the continental US. Geophysical Research Letters, v. 28, n. 10, p. 2077-2080, 2001.

FEDOROVA, N.N.; LEVIT, V.I.; SILVEIRA, M.H.S.; SILVA, B.F.P; AMIRANASHVILI, A.G. Mesoscale convective complexes on the northeastern coast of Brazil. Journal of the Georgian Geophysical Society, v. 13, p. 36-49, 2009.

FERREIRA, A.G.; MELLO, N.G.S. Principais sistemas atmosféricos atuantes sobre a região nordeste do Brasil e a influência dos oceanos pacífico e atlântico no clima da região. Revista Brasileira de Climatologia, v. 1, n. 1, p. 15-28, 2005.

GOMES, H.B.; AMBRIZZI, T.; HERDIES, D.L.; HODGES, K.; SILVA, B.F.P. Easterly Wave Disturbances over Northeast Brazil: An Observational Analysis Advances in Meteorology, v. 2015, ID176238, 2015.

GOOSSENS, C.; BERGER, A. Annual and seasonal climatic variations over the northern hemisphere and Europe during the last century. Annales Geophysicae, Berlin, v. 4, n. B4, p. 385-400, 1986.

HOUNSOU-GBO, G.A.; ARAÚJO, M.; BOURLÉS, B.; VELEDA, D.; SERVAIN, J. Tropical Atlantic Contributions to Strong Rainfall Variability Along the Northeast Brazilian Coast. Hindawi Publishing Corporation Advances in Meteorology, v. 2015, ID902084, p. 1-13, 2015.

HUANG, H.-P.; ROBERTSON, A.W.; KUSHNIR, Y. Atlantic SST gradient and the influence of ENSO. Geophysical Research Letters, v. 32, L20706, 2005a.

IBGE - Instituto Brasileiro de Geografia e Estatística. 2014.

KENDALL, M.G. Rank correlation methods. London, Charles Griffin, 120 p. 1975.

KOUADIO, Y.K.; SERVAIN, J.; MACHADO, L.A.T.; LENTINI, C.A.D. Heavy Rainfall Episodes in Eastern Northeast Brazil Linked to Large-Scale Ocean-Atmosphere Conditions in the Tropical Atlantic. Advances in Meteorology, v. 2012, ID369567, p. 1-16, 2012.

KOUSKY, V.E. Frontal influences on Northeast Brazil. Monthly Weather Review, v. 107, n. 9, p. 1140-1153, 1979.

KOUSKY, V.E.; GAN, M.A. Upper tropospheric cyclonic vortices in the tropical South Atlantic. Tellus, v. 36, n. 6, p. 538-551, 1981.

LYRA, M.J.A.; DA SILVA, N.M.; FEDOROVA, N.; LEVIT, V. Mesoscale convective complexes and thunderstorm events in the Alagoas State, the Northeast Brazil. Lightning Protection (XIII SIPDA), International Symposium, Balneario Camboriu, v. 2015, p. 301-305, 2004. Disponível em $<$ http://ieeexplore.ieee.org/stamp/stamp.jsp?tp=\&arnum ber $=7339305 \&$ isnumber $=7339269>$. Acesso em 27 jun. 2016.

MACEDO, Y.M.; ALMEIDA, L.Q.; TROLEIS, A.L.; MACIEL, A.B.C. Vulnerabilidade socioambiental em escala de detalhe: o caso de Mãe Luiza, Natal, RN, Brasil. Geosaberes, v. 6, n. 3, p. 230-244, 2016.

MANN, H.B. Nonparametric tests against trend. Econometrica, v.13, p. 245-259, 1945.

MARENGO, J.A.; ALVES, L.M.; BEZERRA, E.A.; LACERDA, F.F. Variabilidade e Mudanças climáticas no semiárido brasileiro. INSA - Instituto Nacional do Semiárido. Campina Grande, p. 383-422, 2011.

MARENGO, J.A.; ALVES, L.M.; BESERRA, A.E.; LACERDA, F.F. Variabilidade e mudanças climáticas no semiárido brasileiro, In: Medeiros, S.S.; Gheyi, H.R.; Galvão, C.O.; PAZ, V.P. da S. (orgs), Recursos hídricos em regiões áridas e semiáridas, Campina Grande: INSA, p. 383-422, 2011.

MARENGO, J. O futuro clima do Brasil. Revista USP, v. 103, p. 25-32, 2014.

MATSUURA, K.; WILLMOTT, C.J. Terrestrial Precipitation: 1900-2008 Gridded Monthly Time Series (Version 2.01). Center for Climatic Research Department of Geography University of Delaware Newark, 2009. Disponível em: $<$ http://climate.geog.udel.edu/ climate/html_pages/Glo bal2_Ts_2009/README.global_p_ts_2009.html>. Acesso em 12 out. 2013.

MELO, A.B.C.; CAVALCANTI, I.F.A.; SOUZA, P.P. Zona de convergência intertropical do Atlântico. In: Cavalcanti, I.F.A.; Ferreira, N.J.; Silva, M.G.A.J.; Dias, M.A.F.S (orgs), Tempo e clima no Brasil. São Paulo: Oficina de Textos, p. 25-41, 2009.

MISRA, V.; ZHANG, Y. The fidelity of NCEP CFS seasonal hindcasts over Nordeste. Monthly Weather Review, v. 135, n. 2, p. 618-627, 2007.

MOONEY, P.A.; BRODERICK, C.; BRUYÈRE, C.L.; MULLIGAN, F.J.; PREIN, A.F. Clustering of observed diurnal cycles of precipitation over the United States for evaluation of a WRF multiphysics regional climate ensemble. Journal of Climate, v. 30, n. 22, p. 9267-9286, 2017.

MOREIRA, M.M.; SOUZA, N.M.; CUELLAR, M.D.Z.; ARRAES, K.A. Carta Geotécnica do Município de NatalRN: Áreas de Risco de Deslizamentos e Inundações. In: XVII Congresso Brasileiro de Mecânica dos Solos e Engenharia Geotécnica / VII Congresso Luso-Brasileiro de Geotecnia / VI Simpósio Brasileiro de Mecânica das Rochas / VI Simpósio Brasileiro de Jovens Geotécnicos, Goiânia, 2014.

MOURA, A.D.; SHUKLA, J. On the dynamics of droughts in northeast Brazil: observations, theory and numerical experiments with a general circulation model. Journal of the Atmospheric Sciences, v. 38, n. 12, p. 2653-2675, 1981.

NAMIAS, J. Influence of northern hemisphere general circulation on drought in northeast Brazil. Tellus, v. 24, n. 4, p. 336-342, 1972.

NEWMAN, M.; ALEXANDER, M.A.; AULT, T.R.; COBB, K.M.; DESER, C.; DI LORENZO, E.; MANTUA, N.J.; MILLER, A.J; MINOBE, S.; NAKAMURA, H.; SCHNEIDER, N.; VIMONT, D.J.; PHILLIPS, A.S.; SCOTT, J.D.; SMITH, C.A. The Pacific Decadal Oscillation, Revisited. American Meteor Society, v. 29, n. 12, p. 4399-4427, 2016. 
NICHOLSON, S.E. A semi-quantitative, regional precipitation data set for studying African climates of the nineteenth century, Part I. Overview of the data set. Climate Change, v. 50, n. 1, p. 317-353, 2001.

NÓBREGA, J.N.; DOS SANTOS, C.A.C.; GOMES, O.M.; BEZERRA, B.G.; BRITO, J.I.B. Eventos extremos de precipitação nas mesorregiões da paraíba e suas relações com a tsm dos oceanos tropicais. Revista Brasileira de Meteorologia, v. 29, n. 2, p. 197-208, 2014.

OLIVEIRA, P.T.; SANTOS E SILVA, C.M.; LIMA, K.C. Climatology and trend analysis of extreme precipitation in subregions of Northeast Brazil. Theoretical and Applied Climatology, v. 130, n. 1, p. 77-90, 2017.

OLIVEIRA, P.T.; SILVA, C.M.S.; LIMA, K.C. Linear trend of occurrence and intensity of heavy rainfall events on Northeast Brazil. Atmospheric Science Letters, v. 15, n. 3, p. 172-177, 2014.

OLIVEIRA, P.T.; LIMA, K.C.; SILVA, C.M.S. Synoptic environment associated with heavy rainfall events on the coastland of Northeast Brazil. Advances in Geosciences, v. 35, p. 73-78, 2013.

PETTITT, A.N. A Non-Parametric Approach to the ChangePoint Problem. Applied Statistics, v. 28, n. 2, p. 126-135, 1979.

RAHMAN, M.; YANG, R.; DI, L. Clustering Indian Ocean Tropical Cyclone Tracks by the Standard Deviational Ellipse. Climate, v. 6, n. 39, p. 1-26, 2018.

RAO, V.B.; LIMA, M.C.D.; FRANCHITO, S.H. Sesasonal and interannual variations of rainfall over eastern Northeast Brazil. Journal of Climate, v. 6, n. 9, p. 1754-1763, 1993.

REBOITA, M.S.; GAN, M.A.; ROCHA, R.P.; AMBRIZZI, T. Regimes de precipitação na América do Sul: uma revisão bibliográfica. Revista Brasileira de Meteorologia, v. 25, n. 2, p. 185-204, 2010.

RODRIGUES, D.T.; GONÇALVES, W.A.; SPYRIDES, M.H.C.; SANTOS E SILVA, C.M. Spatial and temporal assessment of the extreme and daily precipitation of the Tropical Rainfall Measuring Mission satellite in Northeast Brazil. International Journal of Remote Sensing, v. 41, n. 2, p. 549-572, 2019.

RODRIGUES, D.T; GONÇALVES, W.A.; SPYRIDES, M.H.C.; SANTOS E SILVA, C.M.; DE SOUZA, D.O. Spatial distribution of the level of return of extreme precipitation events in Northeast Brazil. International Journal of Climatology, v. 41, n. 1, p. 1-16, 2020.

ROGERS, M.J. The association between the North Oscillation and the Southern Oscillation in the Northern Hemisphere. Monthly Weather Review, v. 122, n. 10, p. 1999-2015, 1984.

ROUSSEEUW, P.J. Silhouettes: a Graphical Aid to the Interpretation and Validation of Cluster Analysis. Computational and Applied Mathematics, v. 20, n. 1, p. 53-65, 1987,.

SALEHNIA, N.; SALEHNIA, N.; ANSARI, H.; KOLSOUMI, S.; BANNAYAN, M. Climate data clustering effects on arid and semi-arid rainfed wheat yield: a comparison of artificial intelligence and K-means approaches. International journal of biometeorology, v. 1-12, 2019.
SANTOS E SILVA, C.M.; LUCIO, P., S.; SPYRIDES, M.H.C. Distribuição espacial da precipitação sobre o Rio Grande do Norte: estimativas via satélites e medidas por pluviômetros. Revista Brasileira de Meteorologia, v. 27, n. 3, p. 337-346, 2012.

SANTOS, A.H.M.; ARAGÃO, M.R.S.; CORREIA, M.F.; ARAÚJO, H.A.; SILVA, A.B. Distúrbio Ondulatório de Leste e seus impactos na cidade de Salvador. Revista Brasileira de Meteorologia, v. 27, n. 3, p. 355-364, 2012.

SANTOS, E.B.; LUCIO, S.; SANTOS E SILVA, C.M. Seasonal Analysis of Return Periods for Maximum Daily Precipitation in the Brazilian Amazon. Journal of Hidrometeorology, v. 16, n. 3, p. 973-984, 2015.

SANTOS, N.A.; SIQUEIRA, A.H.B.; MELO, M.L.D. Precipitação sobre a América do Sul e suas relações com a oscilação multidecadal do atlântico e a oscilação do atlântico norte. Revista Brasileira de Climatologia, v. 12, n. 12, p. 207-2018, 2016.

SILVA DOS SANTOS, A.T; SANTOS E SILVA, C.M. Seasonality, Interannual Variability, and Linear Tendency of Wind Speeds in the Northeast Brazil from 1986 to 2011. The Scientific World Journal, v. 2013, ID 490857, 2013.

SMITH, T.M.; REYNOLDS, R.W.; PETERSON, T.C.; LAWRIMORE, J. Improvements to NOAA's Historical Merged Land-Ocean Surface Temperature Analysis (1880-2006). In press. Journal of Climate, v. 21, n. 10, p. 2283-2296, 2007.

SOUZA, E.B.; AMBRIZZI, T. ENSO impacts on the South American rainfall during 1980s: Hadley and Walker circulation. Atmósfera, v. 15, n. 2, p. 105-120, 2002.

SOUZA, E.B.; NOBRE, P. Uma revisão do Padrão Dipolo no Atlântico Tropical. Revista Brasileira de Meteorologia, v.13, n. 1, p. 31-44, 1998.

SOUZA, E.B.; ALVES, J.M.B.; REPELLI, C.A. Um complexo convectivo de mesoescala associado à precipitação intensa sobre Fortaleza-CE. Revista Brasileira de Meteorologia, v.13, n. 2, p. 1-14, 1998.

SOUZA, P; CAVALCANTI, I.F.A. Atmospheric centers of action associated with the Atlantic ITCZ position. Intertional Journal of Climatology, v. 29, n. 14, p. 2091-2105, 2009.

STEFFEN, K.; BOX, J.E.; ABDALATI, W. Greenland Climate Network: GC-Net, US Army Cold Regions Reattach and Engineering (CRREL), CRREL monograph, 1996.

TORRES, R.R.; FERREIRA, N.J. "Case studies of easterly wave disturbances over Northeast Brazil using the Eta Model". Weather and Forecasting, v. 26, n. 2, p. 225-235, 2011.

TRENBERTH, K.E. The definition of El Niño. Bulletin of the American Meteorogical Society, v. 78, n. 12, p. 27712777, 1997.

TRENBERTH, K.; ZHANG, R. National Center for Atmospheric Research Staff (Eds). Last modified 10 Jan 2019. "The Climate Data Guide: Atlantic Multi-decadal Oscillation (AMO)." Disponível no site: https://climatedata guide.ucar.edu/climate-data/atlantic-multi-decadal-oscil lation-amo, acesso em 04 Fev. 2019.

TRZASKA, S.; ROBERTSON, A.W.; FARRARA, J.D.; MECHOSO, C.R. South Atlantic variability arising from air-sea coupling: Local mechanisms and tropical-sub- 
tropical interactions. Journal of Climate, v. 20, n. 14, p. 3345-3365, 2007.

WEBBER, S.R.; WILLMOTT, C.J. South American precipitation: 1960-1990 gridded monthly time series (version 1.02), 1998. Disponível no site: http://climate.geog.udel. edu/ climate/html_pages/README.sa.p.ts.html, acesso em 04 Jun. 2015.

WILCKE, R.A.; BÄRRING, L. Selecting regional climate scenarios for impact modelling studies. Environmental Modelling \& Software, v.78, n. 1, p.191-201, 2016.

WILKS, D.S. Statistical methods in the atmospheric sciences. London: Academic Press, 2006. 648 p.
YAMAZAKI, Y.; RAO, V.B. Tropical cloudiness over the South Atlantic Ocean. Journal of the Meteorological Society of Japan, v. 55, n. 2, p. 205-207, 1977.

ZHOU, J.; LAU, K.M. Principal modes of interannual and decadal variability of summer rainfall over South America. International Journal of Climatology, v.21, n. 13, p. 1623-1644, 2001.

License information: This is an open-access article distributed under the terms of the Creative Commons Attribution License (type CC-BY), which permits unrestricted use, distribution and reproduction in any medium, provided the original article is properly cited. 